\title{
Synchronization of Phase-coupled Oscillators with Plastic Coupling Strength
}

\author{
Andrey Gushchin \\ Center for Applied Mathematics \\ Cornell University \\ Ithaca, NY 14850, USA \\ Email:avg36@cornell.edu
}

\author{
Enrique Mallada \\ Center for the Mathematics \\ of Information \\ California Institute of Technology \\ Pasadena, CA 91125, USA \\ Email: mallada@caltech.edu
}

\author{
Ao Tang \\ School of Electrical \\ and Computer Engineering \\ Cornell University \\ Ithaca, NY 14850, USA \\ Email: atang@ece.cornell.edu
}

\begin{abstract}
In this article we study synchronization of systems of homogeneous phase-coupled oscillators with plastic coupling strengths and arbitrary underlying topology. The dynamics of a coupling strength between two oscillators is governed by the phase difference between these oscillators. We show that such systems are gradient and always achieve frequency synchronization. Moreover, for these systems we provide sufficient stability and instability conditions that are based on results from algebraic graph theory. For a special case when underlying topology is a tree, we formulate a criterion of stability for equilibria. Several examples are used to demonstrate variety of equilibria the system has, and to illustrate differences in behavior of systems with constant and plastic coupling strengths.
\end{abstract}

\section{INTRODUCTION}

Synchronization of phase-coupled oscillators is an extensive topic of research that finds applications in a variety of disciplines including neuroscience ([17], [24], [31], [32]), physics ([24], [4]), mathematics ([10]) and engineering ([8], [23]).

One of the most important properties of each model of phase-coupled oscillators is how coupling between oscillators is defined. First of all, the coupling is characterized by a coupling function, which is a trigonometric $\sin ()$ in case of Kuramoto model - a canonical model for studying synchronization phenomenon. However, a broader class of coupling functions can be considered ([21], [12], [5], [22]). Another characteristic of the coupling is whether coupling strengths are constant or varying with time. While having constant coupling strengths is a more prevalent assumption that generally simplifies analysis of the models, it was suggested ([12], [29], [26]) that considering varying or plastic coupling strengths may be more suitable for studying oscillations in neuroscience, since synaptic neural connections undergo modifications due to learning or forgetting processes.

Synchronization of systems of phase-coupled, and in particular, Kuramoto oscillators has been extensively analyzed under various assumptions: homogeneous ([21]) or heterogeneous ([10], [9]) oscillators; special topology such as complete graph, graph of diameter two or arbitrary ([10], [9]) topology; finite or infinite number of oscillators. However, most of the existing works concentrate on the case of constant coupling strengths. Moreover, those works that study systems of phase-coupled oscillators with plastic coupling strengths generally contain only empirical insights and interesting simulation results. In some cases, analytical description is provided, but only for a few simple special examples such as two connected oscillators [29]. In [28], the authors show some interesting results on synchronization and stability for systems of homogeneous oscillators with plastic coupling strengths, but only for a complete graph topology.

The goal of this work is to develop a general analytical framework for studying systems of phase-coupled homogeneous oscillators with non-constant coupling and arbitrary underlying topology. We show by providing a Lyapunov function, that these systems always achieve frequency synchronization, and derive two sufficient conditions: one for showing stability, and another one for showing instability of equilibrium. Moreover, these conditions characterize all equilibria when underlying topology is a tree graph.

The structure of the article is the following. In the next section we formally describe the model and introduce necessary notation. In Section III we provide several motivating examples. In subsection $A$ of section IV a Lyuponov function is introduced and synchronization of oscillators is shown. Then, in subsection $B$ we formulate stability and instability sufficient conditions. Further, in subsection $C$ we provide a criterion of stability for the tree underlying topology. Finally, in section V we apply our stability results to several examples, and conclude in section VI.

\section{PROBLEM FORMULATION}

We study a system of phase-coupled oscillators with plastic coupling strengths whose dynamics is governed by the following two classes of equations:

$$
\begin{aligned}
& \dot{\phi}_{i}=\omega_{i}+\sum_{j \in N_{i}} K_{i j} \cdot f_{i j}\left(\phi_{j}-\phi_{i}\right), i \in\{1, \ldots, n\} \\
& \dot{K}_{i j}=s_{i j}\left(\alpha_{i j} \cdot F_{i j}\left(\phi_{j}-\phi_{i}\right)-K_{i j}\right), i j \in E,
\end{aligned}
$$

where $E$ is the set of edges. Equation (1a) defines behavior of an oscillator, and equation (1b) determines dynamics of a coupling strength. Here $\phi_{i}$ is a phase of oscillator $i$; $\omega_{i}$ is its intrinsic frequency; $N_{i}$ is a set of oscillators connected to oscillator $i$, i.e. the set of its neighbors; $K_{i j}$ and $f_{i j}$ are a coupling strength and a $2 \pi$-periodic coupling function, 

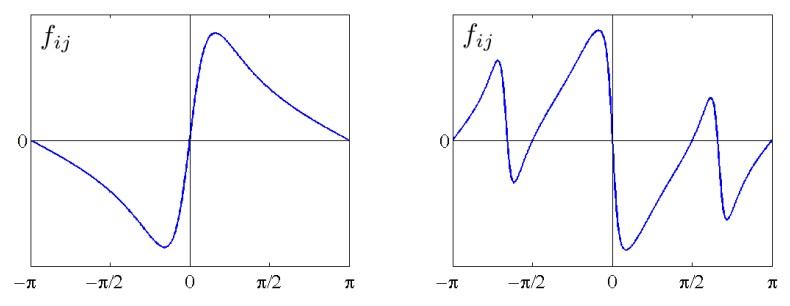

Fig. 1: Examples of function $f_{i j}$ satisfying Assumption 1.

respectively, between connected oscillators $i$ and $j$. In equation (1b), $F_{i j}(x)=-\int_{0}^{x} f_{i j}(t) d t+C$ with a choice of integration constant $C$ that makes $\int_{0}^{\pi} F_{i j}(t) d t=0$. Positive constants $\alpha_{i j}$ determine minimum and maximum values of the coupling strengths, so each $K_{i j}$ takes values from interval $\left[\alpha_{i j} \cdot F_{i j}^{\min }, \alpha_{i j} \cdot F_{i j}^{\max }\right]$, and positive constants $s_{i j}$ define rate of change of the coupling strengths. Notice, that when $f_{i j}\left(\phi_{j}-\phi_{i}\right)=\sin \left(\phi_{j}-\phi_{i}\right) \forall i, j$, then $F_{i j}\left(\phi_{j}-\phi_{i}\right)=$ $\cos \left(\phi_{j}-\phi_{i}\right)$ and system (1) becomes a Kuramoto model with varying coupling strengths which is called a generalized Kuramoto model in [29].

The topology of system (1) is defined by an undirected connected graph $G=(V, E)$ with a vertex set $V$ and an edge set $E$. Each vertex $v_{i} \in V$ corresponds to the oscillator $\phi_{i}$, and each edge $i j \in E$ corresponds to the coupling strength $K_{i j}$, so that $|V|=n$, where $n$ is a number of oscillators in a system, and $N_{i}=\{j \in V \mid i j \in E\}$. Additionally, if oscillators $i$ and $j$ are not connected, then the coupling strength between them is always equal to zero, i.e. $K_{i j} \equiv 0$ if $i j \notin E$. We denote by $m$ the number of edges in a graph so that $|E|=m$. Therefore, the total number of variables and equations in system (1) is $n+m$. It is assumed that coupling is symmetric, and $K_{i j}$ and $K_{j i}$ are the same variable.

In this paper, similarly to [21], we require that functions $f_{i j}$ satisfy these three conditions:

Assumption 1 Functions $f_{i j} \forall i, j$ satisfy:

1) Symmetric coupling: $f_{i j}=f_{j i}$;

2) Odd: $f_{i j}(x)=-f_{i j}(-x)$;

3) $C^{1}: f_{i j}$ is continuously differentiable.

Examples of a $2 \pi$-periodic function $f_{i j}$ satisfying these three conditions are shown on Fig. 1.

To the best of our knowledge, system (1) was initially introduced in [12] as an extension to the classical Kuramoto model of synchronization. Because strength of synapses connections between neurons - can generally change its value and is believed to play a key role in learning and memory formation in the brain, it is natural to consider plastic coupling strengths between oscillators in the Kuramoto model. A well-known synaptic plasticity mechanism called Hebbian rule states that a synapse between two simultaneously active neurons, i.e. neurons that spike almost at the same time, becomes stronger. When neurons are modeled by phase-coupled oscillators, simultaneously firing neurons can be represented by oscillators whose phases are almost equal. This idea is implied in the model (1) with $f_{i j}=\sin ()$, where a connection between two oscillators becomes stronger if it is small enough and if the phases of these oscillators are close to each other. Analytical results presented in our article hold however for a wider set of functions $f_{i j}$ that only need to satisfy Assumption 1.

In the previous works, several modifications of model (1) were introduced and investigated. For example, in [30], [1], [2] time delays are added to the model, and behavior of oscillators for different values of delay parameters is explored. In [20] the coupling strength equation of (1) was replaced by an exponential Spike Timing-Dependent Plasticity (STDP) rule, in which a coupling strength's $K_{i j}$ dependence on a phase difference $\left(\phi_{j}-\phi_{i}\right)$ is defined via exponential function instead of function $f_{i j}^{\prime}$. In [14] a stochastic model of oscillators is studied, where equations (1) contain additive Gaussian noise terms.

In this article we study frequency synchronization of system (1). We say that system (1) achieves frequency synchronization if $\dot{\phi}_{1}(t)=\cdots=\dot{\phi}_{n}(t)=\dot{\phi}$ and $\dot{K}_{i j}(t)=0 \forall i, j$ as $t \rightarrow \infty$, where $\dot{\phi}$ is a common synchronization frequency. We analyze model (1) with homogeneous oscillators which implies that all intrinsic frequencies of oscillators are equal, i.e. there exists a constant $\omega$ such that $\omega_{1}=\cdots=\omega_{n}=\omega$. It is easy to see that if such homogeneous oscillators synchronize, then their synchronization frequency is $\omega$. Without loss of generality, we assume that $\omega=0$, and in the rest of the article consider the following system:

$$
\begin{aligned}
& \dot{\phi}_{i}=\sum_{j \in N_{i}} K_{i j} \cdot f_{i j}\left(\phi_{j}-\phi_{i}\right), \\
& \dot{K}_{i j}=s_{i j}\left(\alpha_{i j} \cdot F_{i j}\left(\phi_{j}-\phi_{i}\right)-K_{i j}\right) .
\end{aligned}
$$

Observe that if $\left(\phi^{*}, K^{*}\right)$ is an equilibrium of system (2), then $\left(\phi^{*}+\delta \mathbf{1}_{n}, K^{*}\right)$, where $\mathbf{1}_{n}$ is a $\mathrm{n}$-dimensional vector of ones and $\delta \in \mathbb{R}$, is also an equilibrium and belongs to the same limit cycle. We will not differentiate between equilibria belonging to the same orbit and thus consider them to be identical. Therefore, in the rest of the article when we talk about stability of an equilibrium $\left(\phi^{*}, K^{*}\right)$, we imply stability of the following set of equilibria: ${ }^{1}$

$$
E_{\phi^{*}}=\left\{(\phi, K)=\left(\phi^{*}+\delta \mathbf{1}_{n}, K^{*}\right), \delta \in \mathbb{R}\right\} .
$$

\section{MOTIVATING EXAMPLES}

In this section we illustrate the differences between the model (2) and the model with constant coupling with several simple examples. The number of oscillators in these examples varies from two to four, and we assume that $f_{i j}\left(\phi_{j}-\phi_{i}\right)=$ $\sin \left(\phi_{j}-\phi_{i}\right)$ for all connected oscillators $i$ and $j$. We demonstrate with these examples that stability of equilibria may change if the coupling strength becomes plastic. Additionally, new equilibria may arise in this case. Moreover, system (2) can possess infinitely many equilibria as shown in Section

\footnotetext{
${ }^{1}$ Alternatively, we could consider phase differences $\left(\phi_{i}-\phi_{j}\right)$ as the variables of system (2) and study stability of a single equilibrium instead of a set (3) of equilibria.
} 

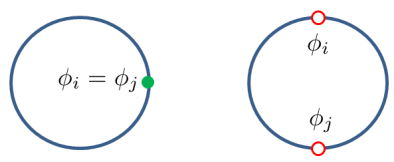

(a) Constant positive coupling strength $K$
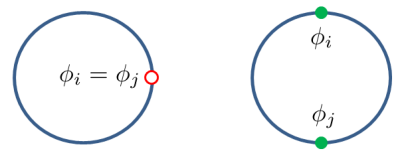

(b) Constant negative coupling strength $K$
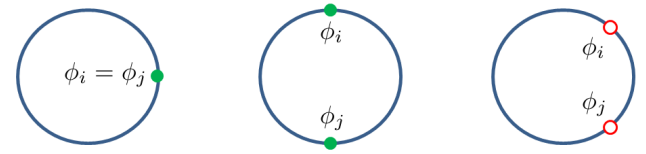

(c) plastic coupling strength $K$

Fig. 2: Example with two oscillators. Green (filled) circles correspond to stable equilibria, red (not filled) circles correspond to unstable equilibria.

III-C. For each example we consider three types of coupling strengths: constant equal positive, constant equal negative, and varying coupling strengths.

\section{A. Two Oscillators}

A system of two connected homogeneous oscillators with a constant positive coupling strength $K_{12}$ and a trigonometric $\sin ()$ coupling function is described by the following equations:

$$
\dot{\phi}_{i}=K_{12} \cdot \sin \left(\phi_{j}-\phi_{i}\right),
$$

where $i=1, j=2$ or $i=2, j=1$. This system has two topologically distinct equilibria: one is in-phase and stable: $\phi_{i}=\phi_{j}$, and another one is anti-phase: $\phi_{j}=\phi_{i}+\pi$ and unstable.

When the coupling strength $K_{12}$ is constant and negative, then the set of equilibria of system (4) remains unchanged, but the in-phase equilibrium now becomes unstable, whereas the anti-phase equilibrium becomes stable.

System (2) of two oscillators with a plastic coupling strength contains two sets of equilibria that are characterized by the following conditions:

1). $\sin \left(\phi_{j}-\phi_{i}\right)=0$. If $\phi_{i}=\phi_{j}$, then $K_{12}=\alpha$, and when $\phi_{j}=\phi_{i}+\pi$, then $K_{12}=-\alpha$.

2). $K_{12}=0$, then $\cos \left(\phi_{j}-\phi_{i}\right)=0$, i.e. $\phi_{j}=\phi_{i}+\pi / 2$. The Jacobian for the system of two oscillators takes form:

$$
J=\left[\begin{array}{ccc}
-K_{12} \cdot \cos () & K_{12} \cdot \cos () & \sin () \\
K_{12} \cdot \cos () & -K_{12} \cdot \cos () & -\sin () \\
\alpha \cdot s \cdot \sin () & -\alpha \cdot s \cdot \sin () & -s
\end{array}\right],
$$

where $\sin ()=\sin \left(\phi_{j}-\phi_{i}\right)$ and $\cos ()=\cos \left(\phi_{j}-\phi_{i}\right)$ for brevity. It can be easily verified that equilibria from the first set are stable, whereas the equilibria from the second set
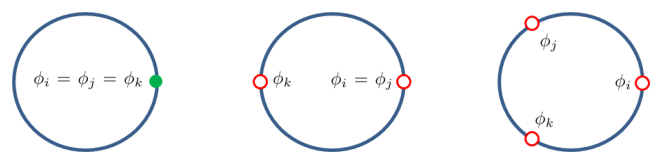

(a) Constant positive coupling strength $K$
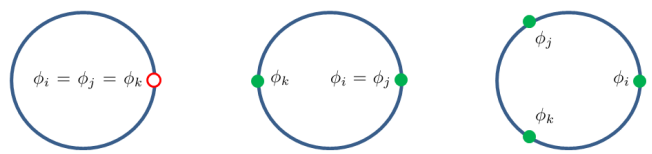

(b) Constant negative coupling strength $K$
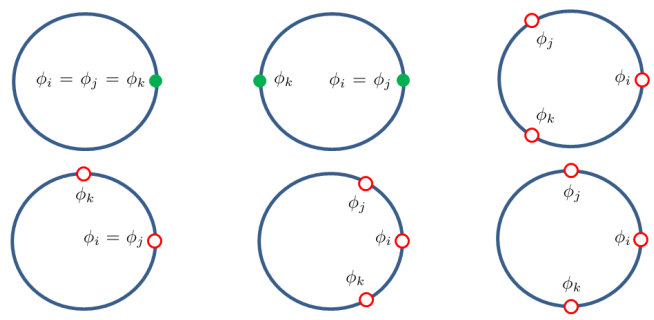

(c) plastic coupling strength $K$

Fig. 3: Example with three oscillators. Green (filled) circles correspond to stable equilibria, red (not filled) circles correspond to unstable equilibria.

(when $K=0$ ) are unstable. Therefore, two observations can be made: all equilibria of system (4) with constant coupling strength are also equilibria of system (2) with plastic coupling strength, and they are all stable for system (2). The second observation is that when the coupling strength is non-constant, a new set of equilibria emerges. This set, however, contains only unstable equilibria in case of two oscillators. On Fig. 3 we depict the sets of equilibria and their stability for three types of coupling described above.

\section{B. Three Oscillators}

In this subsection we consider an example of three connected homogeneous oscillators. We assume that underlying topology is a complete graph, which means that each oscillator is connected to two others. When the coupling function is $\sin ()$ and coupling strength $K$ is constant, the behavior of oscillators is defined by the following set of equations:

$$
\begin{aligned}
& \dot{\phi}_{1}=K \cdot \sin \left(\phi_{2}-\phi_{1}\right)+K \cdot \sin \left(\phi_{3}-\phi_{1}\right), \\
& \dot{\phi}_{2}=K \cdot \sin \left(\phi_{1}-\phi_{2}\right)+K \cdot \sin \left(\phi_{3}-\phi_{2}\right), \\
& \dot{\phi}_{3}=K \cdot \sin \left(\phi_{1}-\phi_{3}\right)+K \cdot \sin \left(\phi_{2}-\phi_{3}\right) .
\end{aligned}
$$

When $K>0$, system (5) has 3 topologically distinct equilibria: one is in-phase when $\phi_{i}=\phi_{j}=\phi_{k}$ and stable, another one is $\phi_{i}=\phi_{j}, \phi_{k}=\phi_{i}+\pi$ and unstable, and the last one is defined as $\phi_{j}=\phi_{i}+2 \pi / 3, \phi_{k}=\phi_{i}-2 \pi / 3$ and is also unstable.

When $K<0$, the set of equilibria of system (5) remains the same. Stability properties of the equilibria, however, change, as in the example with two oscillators. In particular, the first equilibrium becomes unstable, and the last two become stable. 
We now enumerate all equilibria of system (2) with three oscillators assuming that $\alpha_{12}=\alpha_{23}=\alpha_{13}=\alpha$ :

1). In-phase equilibrium: $\phi_{i}=\phi_{j}=\phi_{k}$ and $K_{12}=K_{23}=$ $K_{13}=\alpha$. This equilibrium is stable.

2). $\phi_{i}=\phi_{j}, \phi_{k}=\phi_{i}+\pi$, and $K_{i j}=\alpha, K_{j k}=K_{i k}=-\alpha$, and again a stable equilibrium.

3). $\phi_{j}=\phi_{i}+2 \pi / 3, \phi_{k}=\phi_{i}-2 \pi / 3$, and $K_{i j}=K_{j k}=$ $K_{i k}=-0.5 \cdot \alpha$. This equilibrium is unstable for all positive values of the rate parameter $s>0$. However, when $s=0$, then system (2) becomes a system (5) with constant coupling strengths, and if $K_{i j}=K_{j k}=K_{i k}=-0.5 \cdot \alpha$, this equilibrium is stable.

The equilibria 1), 2) and 3) are also equilibria of system (5), but the following equilibria exclusively correspond to the system (2):

4). $\phi_{i}=\phi_{j}, \phi_{k}=\phi_{i}+\pi / 2$, and the coupling strengths $K_{i j}=\alpha, K_{j k}=K_{i k}=0$. This equilibrium is not stable.

5). $\phi_{j}=\phi_{i}+\pi / 3, \phi_{k}=\phi_{i}-\pi / 3$, and $K_{i j}=K_{i k}=$ $0.5 \cdot \alpha, K_{j k}=-0.5 \cdot \alpha$. Similarly to the equilibrium 3 ), this equilibrium is unstable for all positive values of $s$, and if $s$ is equal to zero while $K_{i j}=K_{i k}=0.5 \cdot \alpha, K_{j k}=-0.5 \cdot \alpha$, then equilibrium is stable.

6). $\phi_{j}=\phi_{i}+\pi / 2, \phi_{k}=\phi_{i}-\pi / 2, K_{i j}=K_{i k}=0$, $K_{j k}=-\alpha$. This is again an unstable equilibrium for all $s>0$, and is stable when $s=0, K_{i j}=K_{i k}=0, K_{j k}=-\alpha$.

Notice that at an equilibrium, $K_{i j}=\cos \left(\phi_{j}-\phi_{i}\right)$ for all $i$ and $j$. If we plug these values of the coupling strengths into the equations of phases, we will obtain:

$$
\begin{aligned}
\sin 2\left(\phi_{2}-\phi_{1}\right)+\sin 2\left(\phi_{3}-\phi_{1}\right) & =0, \\
-\sin 2\left(\phi_{2}-\phi_{1}\right)+\sin 2\left(\phi_{3}-\phi_{2}\right) & =0, \\
\sin 2\left(\phi_{3}-\phi_{1}\right)+\sin 2\left(\phi_{3}-\phi_{2}\right) & =0 .
\end{aligned}
$$

Each of these equations is a direct consequence of the remaining two, and the set of equilibria of system (2) can be found by solving a system of arbitrary two of the above equations.

From the considered examples of two and three oscillators several observations can be made. First, each equilibrium of a system with constant coupling strengths was also an equilibrium of a system with varying coupling strengths. Second, stability of these common equilibria can differ for systems with constant and non-constant coupling. Third, system (2) can possess additional equilibria.

\section{Four Oscillators}

We consider here the case of four oscillators connected by a complete graph. Instead of describing all equilibria of this system, we will show that system (2) with four homogeneous oscillators and $\sin ()$ coupling has infinitely many topologically distinct equilibria.

These equilibria can be defined by means of a parameter $\beta$. Then for each value of $\beta \in(0, \pi / 2)$, phases: $\phi_{j}=\phi_{i}+\pi / 2$, $\phi_{k}=\phi_{i}+\beta, \phi_{l}=\phi_{k}-\pi / 2$, and coupling strengths $K_{i j}=K_{k l}=0, K_{i k}=\cos (\beta), K_{i l}=K_{j k}=\cos (\pi / 2-\beta)=$ $\sin (\beta), K_{j l}=\cos (\pi-\beta)=-\cos (\beta)$ define an equilibrium. Phases corresponding to this equilibrium with a value of parameter $\beta=\pi / 4$ are shown on Fig. 4.

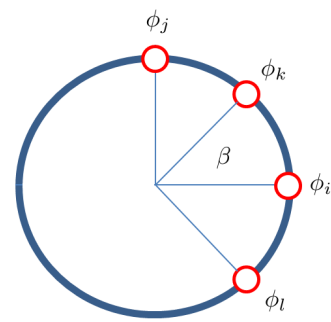

Fig. 4: Equilibrium corresponding to $\beta=\pi / 4$ for the example with four oscillators.

Notice, that in all such equilibria two coupling strengths are equal to zero, and edges corresponding to non-zero coupling strengths form a graph with a ring topology.

These equilibria are unstable for the case of positive learning rate $s$, but if $s=0$, then they become stable.

In several previous works ([13], [15]) a Kuramoto model with dynamic connectivity has been studied. For example, in [13] it was assumed that a system of homogeneous Kuramoto oscillators can learn and remember different patterns, each of which is defined by values of the coupling strengths $K_{i j}$. However, the learning process, i.e. the process of obtaining these patterns was not studied. It was assumed instead that the patterns have been learned and the coupling strengths took the values corresponding to a particular pattern, and were fixed at those values. In addition, only $K= \pm 1$ were considered, and thus only anti-phase equilibria in which some oscillators have phase $\phi$ and the remaining oscillators have phase $\phi+\pi$. There is a finite number of such anti-phase equilibria. In this work we discovered another set of solutions, and the prominent property of this set is that it comprises infinite number of equilibria.

The infinite set of equilibria defined for the case of four oscillators can be generalized for all systems with even $n>2$ number of oscillators. $\phi_{1} \ldots \phi_{\frac{n}{2}-1}$ have the same phase $\phi$, oscillators $\phi_{\frac{n}{2}} \ldots \phi_{n-2}$ have phase $\phi-\frac{\pi}{2}$, and two other oscillators have phases $\phi_{n-1}=\phi-\beta$ and $\phi_{n}=\phi-\beta-\frac{\pi}{2}$.

\section{MAIN RESULTS}

This section is organized as follows. We first show in Theorem 1 by providing a Lyapunov function that system (2) of homogeneous oscillators is gradient and thus always converges to a set of equilibria, i.e. achieves frequency synchronization. After that we formulate sufficient instability and stability conditions for equilibria of system (2) with arbitrary underlying topology in Theorems 2 and 3, respectively. Further, when the underlying topology is a tree and for a particular set of functions $f_{i j}$ that satisfy Assumptions 1 and 2, we derive a criterion of stability and demonstrate that system (2) converges to a stable equilibrium almost surely.

\section{A. Frequency Synchronization}

In Theorem 1 we prove that system (2) of homogeneous oscillators is a gradient system and always achieves frequency synchronization. A similar result was obtained in [28], where 
a potential function was found for system (2) and frequency synchronization was shown, but only for the case of a complete graph topology. Although the potential function is not really different in the case of an arbitrary topology, we provide a proof of Theorem 1 for completeness.

Theorem 1 (Frequency synchronization) System (2) is a gradient system and achieves frequency synchronization for all initial values of phases and coupling strengths.

Proof. Notice, that all variables of system (2): $\phi_{i}, K_{i j}$ are defined on a compact set. Indeed, all phase variables $\phi_{i}$ are defined on a n-dimensional torus $T^{n}$, and all coupling variables $K_{i j}$ are defined on $\left[\alpha_{i j} \cdot F_{i j}^{\min }, \alpha_{i j} \cdot F_{i j}^{\max }\right]^{\frac{n(n-1)}{2}}$. We can also provide a potential function $V$ :

$$
V=-\sum_{i j \in E, i>j} K_{i j} F_{i j}\left(\phi_{i}-\phi_{j}\right)+\frac{1}{2} \sum_{i j \in E, i>j} \frac{K_{i j}^{2}}{\alpha_{i j}} .
$$

This function is well-defined, bounded, and it is easy to verify that the derivative of $V$ is:

$$
\dot{V}=(\nabla V)^{T} *\left[\begin{array}{c}
\dot{\phi}_{i} \\
\cdot \\
\dot{\phi}_{n} \\
\dot{K}_{21} \\
\cdot \\
\cdot \\
\dot{K}_{n, n-1}
\end{array}\right]=-\sum_{i=1}^{n}\left(\dot{\phi}_{i}\right)^{2}-\sum_{i j \in E, i>j} \frac{\left(\dot{K}_{i j}\right)^{2}}{\alpha_{i j} s_{i j}} .
$$

We can see that $\dot{V}$ is always non-positive and is equal to zero if and only if $\dot{\phi}_{i}=0$ and $\dot{K}_{i j}=0$ for all $i$ and $j$. Thus, by LaSalle's Invariance Principle [19], the trajectories of (2) always converge to a set of equilibria. In other words, for all initial conditions frequency synchronization occurs.

Remark Notice that Theorem 1 does not imply pointwise convergence to a single equilibrium. It is also not guaranteed that equilibria of system (2) are isolated.

\section{B. Stability And Instability Conditions For Arbitrary Topology}

The main results of this subsection are Theorem 2 which is a sufficient instability condition, and Theorem 3 that defines a sufficient condition for stability of an equilibrium of system (2). These results are based on Lyapunov's indirect method [16], that states:

1) If $\operatorname{Re} \lambda_{i}<0$ for all eigenvalues of the Jacobian matrix $J$, then equilibrium is asymptotically stable.

2) If $\operatorname{Re} \lambda_{i}>0$ for at least one eigenvalue of the Jacobian matrix $J$, then equilibrium is unstable.

Let $B \in \mathbb{R}^{n \times m}$ denote an oriented incidence matrix of a graph that defines underlying topology of system (2). Then element $(i, e)$ of this matrix is

$$
B(i, e)= \begin{cases}1 & \text { if } i \text { is the head of } e \\ -1 & \text { if } i \text { is the tail of } e \\ 0 & \text { otherwise. }\end{cases}
$$

Although the definition of matrix $B$ implies that graph $G$ is oriented, all properties of this matrix used in this article do not depend on a particular orientation. Therefore, we assume that for a given undirected graph $G$, an arbitrary orientation of its edges is chosen, i.e. for every undirected edge $e=(u, v)$ one of the nodes $u, v$ is designated as a head of $e$, and another one corresponds to a tail of $e$.

Let $\alpha \in \mathbb{R}^{m}, s \in \mathbb{R}^{m}, K \in \mathbb{R}^{m}, f^{\prime} \in \mathbb{R}^{m}$ and $f \in \mathbb{R}^{m}$ denote vectors whose components are $\alpha_{i j}, s_{i j}, K_{i j}, f_{i j}^{\prime}\left(\phi_{j}-\right.$ $\left.\phi_{i}\right)$ and $f_{i j}\left(\phi_{j}-\phi_{i}\right)$, respectively, for each $i, j$ such that $i j \in E$. We will use symbol $*$ to denote the componentwise product of vectors. Jacobian of system (2) can be written in a following way:

$$
J=\left[\begin{array}{cc}
B & 0 \\
0 & I
\end{array}\right]\left[\begin{array}{cc}
-\operatorname{diag}\left(K * f^{\prime}\right) & -\operatorname{diag}(f) \\
-\operatorname{diag}(\alpha * s * f) & -\operatorname{diag}(s)
\end{array}\right]\left[\begin{array}{cc}
B^{T} & 0 \\
0 & I
\end{array}\right],
$$

The first matrix in a product is of size $(n+m) \cdot(m+m)$, the second matrix is of size $(m+m) \cdot(m+m)$ and the last matrix in the product has dimensions $(m+m) \cdot(n+m)$. Notice that Jacobian $J$ has a trivial eigenvector $\left[\begin{array}{ll}\mathbf{1}_{n} & \mathbf{0}_{m}\end{array}\right]^{T}$, where $\mathbf{1}_{n} \in \mathbb{R}^{n}$ is a vector of ones and $\mathbf{0}_{m} \in \mathbb{R}^{m}$ is a vector of zeros with $n$ and $m$ components, respectively. This eigenvector emerges due to rotational invariance of system (2) and corresponds to a zero eigenvalue. Since trajectories of system (2) are orthogonal to the direction of an orbit, we still can apply Lyapunov's indirect method to explore stability of the set (3). If all remaining eigenvalues of the Jacobian have negative real part, then equilibrium is stable; if there exists an eigenvalue with a positive real part, then equilibrium is unstable.

The component of vector $f$ that corresponds to the edge $e=(i, j)$ is equal to $f_{i j}\left(\phi_{i}-\phi_{j}\right)$ if edge $e=(i, j)$ is oriented from a tail $j$ to a head $i$, and thus $B(i, e)=1, B(j, e)=-1$. Similarly, if edge $e=(i, j)$ is oriented from a tail $i$ to a head $j$, then $B(i, e)=-1, B(j, e)=1$ and component of $f$ associated with edge $i j$ is equal to $f_{i j}\left(\phi_{j}-\phi_{i}\right)$.

Each partition $P$ of the graph's vertices into two sets $V^{-}$ and $V^{+}$such that $V^{-} \cap V^{+}=\emptyset$ and $V^{-} \cup V^{+}=V$, defines a cut $C(P) \triangleq\left\{i j \in E \mid i \in V^{-}, j \in V^{+}\right\}$. With each cut $C(P)$ we associate a cut vector $c_{P} \in \mathbb{R}^{m}$ which is defined as follows:

$$
c_{P}(e)= \begin{cases}1 & \text { if } e \text { goes from } V^{-} \text {to } V^{+}, \\ -1 & \text { if } e \text { goes from } V^{+} \text {to } V^{-}, \\ 0 & \text { if } e \notin C(P) .\end{cases}
$$

We can now formulate the following instabiliy condition that is similar to Theorem 2 of [21]:

Theorem 2 (Sufficient instability condition) If there exists a cut $C(P)$ such that at equilibrium $\left(\phi^{*}, K^{*}\right)$ of system (2):

$$
\sum_{i j \in C(P)}\left(K_{i j} f_{i j}^{\prime}-\alpha_{i j} f_{i j}^{2}\right)<0,
$$

where $K_{i j}=K_{i j}^{*}, f_{i j}^{\prime}=f_{i j}^{\prime}\left(\phi_{j}^{*}-\phi_{i}^{*}\right)$ and $f_{i j}=f_{i j}\left(\phi_{j}^{*}-\phi_{i}^{*}\right)$, then $\left(\phi^{*}, K^{*}\right)$ is an unstable equilibrium. 
Proof. We first show that the Jacobian of system (2) can be decomposed into a product of matrices $D$ and $A$ :

$$
J=D A,
$$

where $D$ is a positively-definite diagonal matrix, and $A$ is a symmetric matrix. We then demonstrate that stability of equilibria of system (2) does not depend on matrix $D$, because matrices $J$ and $A$ have the same number of positive, negative and zero eigenvalues. Next, for matrix $A$ we provide a vector $\vec{X}$ such that $\vec{X}^{T} A \vec{X}>0$, which guarantees that the symmetric matrix $A$ has a positive eigenvalue and so does the Jacobian matrix $J$. This in turn means that an equilibrium is unstable due to Lyapunov's indirect method.

Decomposition (10) is possible because system (2) is a gradient system. Note that the Hessian matrix $H(V)$ of the potential function $V$ is symmetric. Let matrix $D$ be defined as

$$
D=\left[\begin{array}{cc}
I & 0 \\
0 & \operatorname{diag}(\alpha * s)
\end{array}\right],
$$

then, since equations (2) can be written as follows:

$$
\left[\begin{array}{c}
\dot{\phi} \\
\dot{K}
\end{array}\right]=-D \cdot \nabla V,
$$

decomposition (10) exists with $A=-H(V)$.

We now show that matrices $J$ and $A=-H(V)$ have the same numbers of positive, negative and zero eigenvalues. Observe that if matrix $D^{\frac{1}{2}}$ is a square root of matrix $D$, then matrices $D A$ and $D^{\frac{1}{2}} A D^{\frac{1}{2}}$ have the same eigenvalues, because matrix $D$ is positive-definite. This also implies that Jacobian of system (2) with homogeneous oscillators has only real eigenvalues. Next, since $A$ is a symmetric matrix with real entries, it can be diagonalized by an orthogonal matrix, i.e. there exists a real orthogonal matrix $Q$ such that $A=Q G Q^{T}$, where $G$ is a diagonal matrix. Further, notice that

$$
D^{\frac{1}{2}} A D^{\frac{1}{2}}=D^{\frac{1}{2}} Q G Q^{T} D^{\frac{1}{2}}=L G L^{T},
$$

where matrix $L$ is defined as $L=D^{\frac{1}{2}} Q$ and is invertible. Therefore,

$$
Q^{T} A Q=L^{-1}\left(D^{\frac{1}{2}} A D^{\frac{1}{2}}\right)\left(L^{-1}\right)^{T}=G .
$$

By Sylvester's law of inertia, numbers of positive, negative and zero eigenvalues of matrices $A, D^{\frac{1}{2}} A D^{\frac{1}{2}}$ and $G$ are equal. Thus, since $J=D A$ and $D^{\frac{1}{2}} A D^{\frac{1}{2}}$ have equal eigenvalues, numbers of positive, negative and zero eigenvalues of matrices $J$ and $A$ are the same.

We now consider the symmetric matrix $A$ and show that when condition (9) is satisfied, matrix $A$ has a positive eigenvalue. We define a matrix $M$ to be:

$$
M=\left[\begin{array}{cc}
\operatorname{diag}\left(K * f^{\prime}\right) & \operatorname{diag}(f) \\
\operatorname{diag}(f) & \operatorname{diag}(1 / \alpha)
\end{array}\right],
$$

where $1 / \alpha$ is a vector with components $1 / \alpha_{i j}$, then

$$
A=-\left[\begin{array}{cc}
B & 0 \\
0 & I
\end{array}\right] M\left[\begin{array}{cc}
B^{T} & 0 \\
0 & I
\end{array}\right] \text {. }
$$

We denote:

$$
\hat{B}=\left[\begin{array}{cc}
B & 0 \\
0 & I
\end{array}\right]
$$

and then

$$
A=-\hat{B} M \hat{B}^{T}
$$

Now we will assume that there exists a cut $C(P)$ that satisfies condition (9). We define a vector $\vec{Y} \in R^{2 m}$ to be:

$$
\vec{Y}=\left[\begin{array}{c}
c_{P} \\
-c_{P} * f * \alpha
\end{array}\right],
$$

where $c_{P}$ is a cut vector associated with the cut $C(P)$, and multiplication in $-c_{P} * f * \alpha$ is componentwise.

It can be verified that the sum from (9) is equal to $\vec{Y}^{T} M \vec{Y}$. Indeed, if an edge $k(1 \leq k \leq m)$ belongs to the cut $C(P)$, then $\vec{Y}_{k}= \pm 1$ and $\vec{Y}_{k+m}=\mp f_{k} \alpha_{k}$. The summand number $k$ in $\vec{Y}^{T} M \vec{Y}$ is equal to:

$$
\begin{aligned}
& Y_{k}^{2} K_{k} f_{k}^{\prime}+2 Y_{k} Y_{m+k} f_{k}+\frac{Y_{m+k}^{2}}{\alpha_{k}} \\
& =K_{k} f_{k}^{\prime}-2 f_{k}^{2} \alpha_{k}+f_{k}^{2} \alpha_{k}=K_{k} f_{k}^{\prime}-f_{k}^{2} \alpha_{k},
\end{aligned}
$$

which is also the $k^{\text {th }}$ summand of the sum (9).

The cut space of graph $G$ is defined as a space spanned by all cut vectors $c_{P}$. It is known (see for example [3]) that the range of $B^{T}$ is the cut space of $G$. Therefore, for the cut vector $c_{P}$ there exists a vector $\vec{x}_{1} \in R^{n}$ such that $c_{P}=B^{T} \vec{x}_{1}$. Therefore,

$$
\vec{Y}=\left[\begin{array}{c}
B^{T} \vec{x}_{1} \\
-c_{P} * f * \alpha
\end{array}\right]=\hat{B}^{T} \vec{X},
$$

where $\vec{X}=\left[\begin{array}{c}\vec{x}_{1} \\ -c_{P} * f * \alpha\end{array}\right] \in R^{n+m}$.

Finally,

$$
0>\vec{Y}^{T} M \vec{Y}=\vec{X}^{T} \hat{B} M \hat{B}^{T} \vec{X}=-\vec{X}^{T} A \vec{X},
$$

which means that their is a vector $\vec{X}$ such that $\vec{X}^{T} A \vec{X}>0$ and thus symmetric matrix $A$ has a positive eigenvalue which implies that Jacobian $J$ has also a positive eigenvalue. Therefore, equilibrium $\left(\phi^{*}, K^{*}\right)$ is unstable.

We now formulate a sufficient condition for an equilibrium of system (2) to be stable.

Theorem 3 (Sufficient stability condition) If at equilibrium $\left(\phi^{*}, K^{*}\right)$ of system (2), for each ij $\in E$ :

$$
K_{i j} f_{i j}^{\prime}-\alpha_{i j} f_{i j}^{2}>0
$$

where $K_{i j}=K_{i j}^{*}, f_{i j}^{\prime}=f_{i j}^{\prime}\left(\phi_{j}^{*}-\phi_{i}^{*}\right)$, then equilibrium $\left(\phi^{*}, K^{*}\right)$ is asymptotically stable.

Proof. All eigenvalues of the Jacobian of system (2) are real. To apply Lyapunov's indirect method, we need to show that at equilibrium $\left(\phi^{*}, K^{*}\right)$ Jacobian has only negative eigenvalues. However, it has always at least one zero eigenvalue that corresponds to the rotational invariance of the system: if all phases $\phi_{i}(i=1, \ldots, n)$ are simultaneously shifted by the same value, system does not change. The eigenvector associated with this zero eigenvalue is a vector $\left[\begin{array}{ll}\mathbf{1}_{n} & \mathbf{0}_{m}\end{array}\right]^{T}$. 
In this article we do not distinguish equilibria that belong to the same set (3), and study stability of the whole set $E_{\phi^{*}}$. As was previously mentioned, to show stability of $E_{\phi^{*}}$ using an indirect Lyapunov's method, we need to show that all remaining eigenvalues of the Jacobian are strictly negative.

In the proof of Theorem 2 it was shown that the Jacobian matrix $J$ and symmetric matrix $A$ have the same numbers of negative, positive and zero eigenvalues. This means that matrix $A$ also possesses a zero eigenvalue corresponding to the rotational invariance. Moreover, it is easy to see that vector $\left[\begin{array}{ll}\mathbf{1}_{n} & \mathbf{0}_{m}\end{array}\right]^{T}$ is also an eigenvector of matrix $A$ associated with a zero eigenvalue. Therefore, to prove that equilibrium $\left(\phi^{*}, K^{*}\right)$ is stable, it is sufficient to demonstrate that all eigenvalues of matrix $A$ are negative (except for one zero eigenvalue corresponding to the rotational invariance), or that $\vec{X}^{T} A \vec{X}<0$ for all non-zero vectors $\vec{X} \in \mathbb{R}^{n+m}, \vec{X} \neq \operatorname{span}\left(\left[\mathbf{1}_{n} \mathbf{0}_{m}\right]^{T}\right)$, since $A$ is symmetric.

Notice that because $A=-\hat{B} M \hat{B}^{T}$, matrix $A$ will have only negative eigenvalues (except one) if $\vec{Y}^{T} M \vec{Y}>0$ for all non-zero vectors $\vec{Y} \in \mathbb{R}^{2 m}$. Indeed, if $\hat{B}^{T} \vec{X} \neq \mathbf{0}_{n+m}$, then

$$
\vec{X}^{T} A \vec{X}=-\vec{X}^{T} \hat{B} M \hat{B}^{T} \vec{X}=-\vec{Y}^{T} M \vec{Y}<0,
$$

where vector $\vec{Y} \triangleq \hat{B}^{T} \vec{X}$.

Additionally, if $\vec{X}=\left[\begin{array}{ll}\vec{x}_{1} & \vec{x}_{2}\end{array}\right]^{T}$, where $\vec{x}_{1}$ are the first $n$ components of $\vec{X}$, and $\vec{x}_{2}$ are the last $m$ components of $\vec{X}$, then $\hat{B}^{T} \vec{X}=\mathbf{0}_{n+m}$ only if $B^{T} \vec{x}_{1}=\mathbf{0}_{n}$ and $\vec{x}_{2}=\mathbf{0}_{m}$. And since $\operatorname{ker}\left(B^{T}\right)=\operatorname{span}\left(\mathbf{1}_{n}\right)$ for a connected $G$ (see for example [3]), then $\hat{B}^{T} \vec{X} \neq \mathbf{0}_{n+m}$ if $\vec{X} \neq \operatorname{span}\left(\left[\begin{array}{ll}\mathbf{1}_{n} & \mathbf{0}_{m}\end{array}\right]^{T}\right)$.

Therefore, it is now enough to show that condition (23) is sufficient for matrix $M$ to be positively definite. Let $\vec{Y} \in \mathbb{R}^{2 m}$ be an arbitrary vector, then $\vec{Y}^{T} M \vec{Y}$ is a sum of $m$ terms, where the $k^{\text {th }}$ term is equal to

$$
Y_{k}^{2} K_{k} f_{k}^{\prime}+2 Y_{k} Y_{m+k} f_{k}+\frac{Y_{m+k}^{2}}{\alpha_{k}} .
$$

We now consider this term as a quadratic function of $Y_{k}$. This equation is an equation of parabola whose branches are directed upwards because $K_{k} f_{k}^{\prime}>0$ due to (23). Then, the minimum value of (25) is achieved at the vertex of the parabola and is equal to:

$$
\begin{aligned}
& \left(\frac{Y_{m+k} f_{k}}{K_{k} f_{k}^{\prime}}\right)^{2} K_{k} f_{k}^{\prime}-2\left(\frac{Y_{m+k} f_{k}}{K_{k} f_{k}^{\prime}}\right) Y_{m+k} f_{k}+\frac{Y_{m+k}^{2}}{\alpha_{k}} \\
& =-\frac{Y_{m+k}^{2} f_{k}^{2}}{K_{k} f_{k}^{\prime}}+\frac{Y_{m+k}^{2}}{\alpha_{k}}=Y_{m+k}^{2}\left(-\frac{f_{k}^{2}}{K_{k} f_{k}^{\prime}}+\frac{1}{\alpha_{k}}\right) .
\end{aligned}
$$

The last expression is positive if $Y_{m+1} \neq 0$ and if condition (23) is satisfied.

Suppose that $Y_{m+k}=0$, then (25) becomes equal to $Y_{k}^{2} K_{k} f_{k}^{\prime} \geq 0$, and is equal to zero only if $Y_{k}=0$. Since $\vec{Y}$ is a non-zero vector, there exists at least one component $k$ of vector $\vec{Y}$ such that the sum (25) is strictly positive, and for all other components these sums are non-negative. Therefore, for all vectors $\vec{Y} \in \mathbb{R}^{2 m}: \vec{Y}^{T} M \vec{Y}>0$, and $\vec{X}^{T} A \vec{X}<0$ for all vectors $\vec{X} \in \mathbb{R}^{n+m}$ such that $\vec{X} \neq \operatorname{span}\left(\left[\begin{array}{ll}\mathbf{1}_{n} & \mathbf{0}_{m}\end{array}\right]^{T}\right)$. Thus, all eigenvalues of $A$ except one are negative, so are eigenvalues of $J$, and equilibrium $\left(\phi^{*}, K^{*}\right)$ is asymptotically stable.
Remark Notice that conditions (9) and (23) can be written as follows:

$$
\begin{array}{r}
\sum_{i j \in C(P)}\left(F_{i j} f_{i j}^{\prime}-f_{i j}^{2}\right)<0, \\
F_{i j} f_{i j}^{\prime}-f_{i j}^{2}>0,
\end{array}
$$

because at equilibrium: $K_{i j}=\alpha_{i j} F_{i j}$ and $\alpha_{i j}>0$. Thus, they are independent of $\alpha_{i j}$.

\section{Stability And Instability Conditions For Tree Topology}

In this subsection we consider system (2) of homogeneous oscillators when the underlying topology graph $G$ is a tree. For example, star and chain graphs are the graphs with a tree topology. When the topology is a tree, each single edge defines a cut of $G$, and using Theorem 2, a sufficient instability condition for tree graphs can be formulated as follows:

Corollary 4 (Sufficient instability condition for trees) If there exists an edge $i j \in E$ such that at equilibrium $\left(\phi^{*}, K^{*}\right)$ of system (2) with tree topology:

$$
K_{i j} f_{i j}^{\prime}-\alpha_{i j} f_{i j}^{2}<0,
$$

where $K_{i j}=K_{i j}^{*}$, $f_{i j}^{\prime}=f_{i j}^{\prime}\left(\phi_{j}^{*}-\phi_{i}^{*}\right)$ and $f_{i j}=f_{i j}\left(\phi_{j}^{*}-\phi_{i}^{*}\right)$, then $\left(\phi^{*}, K^{*}\right)$ is an unstable equilibrium.

Using Theorem 3 and Corollary 4, stability of an equilibrium of system (2) with a tree topology can be determined if $K_{i j} f_{i j}^{\prime}-\alpha_{i j} f_{i j}^{2} \neq 0$ for every $i j \in E$. To guarantee that the last condition is always satisfied, we will now concentrate on a more special class of functions $f_{i j}()$. In particular, these functions must fulfill the following conditions.

Assumption 2 Functions $f_{i j} \forall i, j$ satisfy:

1) Assumption 1;

2) $f_{i j}^{\prime}(0)>0, f_{i j}^{\prime}(\pi)<0$;

3) $f_{i j}(x)>0, \forall x \in(0, \pi)$,

Example of a function that meets all conditions of Assumption 2 is shown on the left side of Fig. 1. The following fact establishes a property of all equilibria of system (2) with a tree topology, and with functions $f_{i j}()$ satisfying Assumption 2.

Lemma 5 Let $\left(\phi^{*}, K^{*}\right)$ be an equilibrium of system (2) with a tree underlying topology and with functions $f_{i j}()$ satisfying Assumption 2, and let $f_{i j}^{*} \triangleq f_{i j}\left(\phi_{j}^{*}-\phi_{i}^{*}\right) \forall i, j$. Then for each pair $i j \in E$, exactly one of the following two conditions is satisfied:

- $f_{i j}^{*}=0$; this implies that either $\phi_{j}^{*}-\phi_{i}^{*}=0$, or $\phi_{j}^{*}-\phi_{i}^{*}=\pi$.

- $K_{i j}^{*}=0$; this implies that $F_{i j}^{*}=0$.

Proof. Since the underlying topology is defined by a graph $G$ that is a tree, there are nodes in $G$ each of which has a single neighbor. These nodes are the leaves of a tree graph $G$. Let $\phi_{i}$ be an oscillator associated with a leaf node $i$, and let $\phi_{j}$ be an oscillator such that node $j$ is a single neighbor of node $i$. Then, from equation (1a), at an equilibrium $\left(\phi^{*}, K^{*}\right): \dot{\phi}_{i}=0$ if and only if $K_{i j}^{*}=0$ or $f_{i j}\left(\phi_{j}^{*}-\phi_{i}^{*}\right)=0$. Because $K_{i j}^{*}=0$ if and only if $F_{i j}^{*}=0$, and for function $f_{i j}$ satisfying Assumption 2, $f_{i j}$ and $F_{i j}$ are not equal to zero simultaneously, either $f_{i j}^{*}=0$ 
or $K_{i j}^{*}=F_{i j}^{*}=0$. We then remove all leaf nodes from the graph $G$ and apply the same reasoning for the leaves of a new smaller graph which is also a tree. We repeat this procedure until we obtain a single node, and at each step condition of the theorem is satisfied.

Corollary 6 For tree topology all equilibria are isolated.

We can now see that when the underlying topology of system (2) is a tree, and if coupling functions $f_{i j}()$ satisfy Assumption 2, then at equilibrium:

$$
K_{i j} f_{i j}^{\prime}-\alpha_{i j} f_{i j}^{2} \neq 0
$$

for every $i j \in E$. Indeed, if at equilibrium $f_{i j}=0$, then from Lemma $5, K_{i j} \neq 0$, and $f_{i j}^{\prime} \neq 0$ due to Assumption 2. If $K_{i j}=0$, i.e. $F_{i j}=0$, then by definition of $F_{i j}$ and from Assumption 2: $f_{i j} \neq 0$.

Since Assumption 2 guarantees that condition (29) holds for every equilibrium, it is possible to formulate a criterion of stability for system (2) whose underlying topology is a tree graph.

Theorem 7 (Criterion of stability for the tree topology) If the underlying topology of system (2) is a tree, and functions $f_{i j}()$ satisfy Assumption 2 for each $i j \in E$, then an equilibrium $\left(\phi^{*}, K^{*}\right)$ is stable if and only if condition (23) holds for every edge $i j \in E$.

We now provide a result regarding ranks of matrices $\hat{B}$ and $M$ for a tree topology.

Lemma 8 For tree topology:

$$
\begin{aligned}
n & =m+1, \\
\operatorname{Rank}(\hat{B})=\operatorname{Rank}\left(\hat{B}^{T}\right) & =\min (n+m, m+m)=2 m, \\
\operatorname{Rank}(M) & =2 m .
\end{aligned}
$$

Proof. The first equation says that in a tree graph the number of vertices is greater than the number of edges by one, and is obvious. Second fact follows from the properties of the incidence matrix $B$ and because we consider a tree topology. We now show that the third equation is correct. Recall that matrix $M$ has dimensions $(2 m) \cdot(2 m)$. Suppose by contradiction that $M$ has a zero eigenvalue, then there exists a non-zero vector $\vec{x}$ such that $M \vec{x}=0$. This system contains $2 m$ equations, the first $m$ of them are of the form:

$$
x_{i} K_{i} f_{i}^{\prime}+x_{m+i} f_{i}=0,
$$

where $i=1, \ldots, m$, and the remaining $m$ equations are:

$$
x_{i} \cdot f_{i}+x_{m+i} / \alpha_{i}=0,
$$

where $i=1, \ldots, m$ again. We choose a particular index $i$ such that $1 \leq i \leq m$, and consider a pair of corresponding equations: one from (30) and another one from (31). We now use Lemma 5 and first consider the case when $f_{i}^{*}=0$. Then, from the first equation, $x_{i}=0$ because $K_{i} \neq 0$ and $f_{i}^{\prime} \neq 0$. And from the second equation: $x_{m+i}=0$ since $\alpha_{i}>0$. Now consider the case when $K_{i}^{*}=0$, then from the first equation: $x_{m+i}=0$, and then from the second equation: $x_{i}=0$ since $f_{i} \neq 0$. Therefore, in both cases $x_{i}=x_{m+i}=0$, and since this should be true for all $1 \leq i \leq m$, vector $\vec{x}$ has to be a zero vector which contradicts our assumption that $\vec{x}$ is nonzero.

While Theorem 1 does not guarantee pointwise convergence and isolation of equilibria in general, for the case of a tree underlying topology we proved isolation of equilibria in Lemma 5, and now can show that it converges to a stable equilibrium almost surely.

Theorem 9 At any equilibrium of system (2) with a tree topology and with functions $f_{i j}$ satisfying Assumption 2, Jacobian has only one zero eigenvalue due to rotational invariance, and system converges to a stable equilibrium almost surely.

Proof. Jacobian matrix is of size $(n+m) \cdot(n+m)$ or, using Lemma 8 , of size $(2 m+1) \cdot(2 m+1)$, and can be expressed as follows:

$$
J=-\hat{B} M \hat{B}^{T},
$$

and matrix $M$ is of size $(2 m) \cdot(2 m)$, matrices $\hat{B}$ and $\hat{B}^{T}$ are of size $(2 m+1) \cdot(2 m),(2 m) \cdot(2 m+1)$, respectively. We now employ the following fact: if matrix $A$ has dimensions $x \cdot y$, matrix $B$ is of size $y \cdot z$ and $\operatorname{Rank}(B)=y$, then $\operatorname{Rank}(A B)=\operatorname{Rank}(A)$. Using this fact we conclude that $\operatorname{Rank}(\hat{B} M)=2 m$, and $\operatorname{Rank}(J)=\operatorname{Rank}\left(\hat{B} M \hat{B}^{T}\right)=2 m$. Since rank of a matrix is equal to the number of non-zero eigenvalues, Jacobian matrix $J$ has only one zero eigenvalue which is due to the rotational invariance. Thus, all equilibria of (2) with tree topology and functions $f_{i j}$ satisfying Assumption 2 , are hyperbolic when domain of the system is restricted to the subspace orthogonal to $\left[\begin{array}{ll}\mathbf{1}_{n} & \mathbf{0}_{m}\end{array}\right]^{T}$. Therefore, system (2) almost surely converges to a stable equilibrium.

\section{NUMERICAL ILLUSTRATIONS}

In this section we consider several network examples and apply our results to explore stability of their equilibria. In these example we will assume that $f_{i j}=\sin \left(\phi_{j}-\phi_{i}\right)$ and $F_{i j}=-\cos \left(\phi_{j}-\phi_{i}\right)$, and system (2) becomes a generalized Kuramoto model with plastic coupling strengths. Notice that this choice of functions $f_{i j}$ guarantees that Assumption 2 is satisfied.

\section{A. Two Oscillators}

Since two connected oscillators form a tree topology, we can apply a criterion of stability provided in Theorem 7 . We will assume that $\alpha_{12}$ takes an arbitrary positive value.

In subsection III-A we described two types of equilibria of the system of two oscillators. The first type corresponds to the case when $\sin \left(\phi_{2}-\phi_{1}\right)=0$, which implies that $f_{12}=0$, $K_{12} / \alpha_{12}=f_{12}^{\prime}=\cos \left(\phi_{2}-\phi_{1}\right)$ and sufficient condition (23) is satisfied making these equilibria stable.

The second type of equilibria for this system is when $\cos \left(\phi_{2}-\phi_{1}\right)=0$, i.e. $K_{12}=0$, and $f_{12}=\sin \left(\phi_{2}-\phi_{1}\right)=1$. Then, inequality (28) holds and equilibrium is unstable.

Behavior of a system of two oscillators after a small perturbation from the anti-phase equilibrium is shown on Fig. 


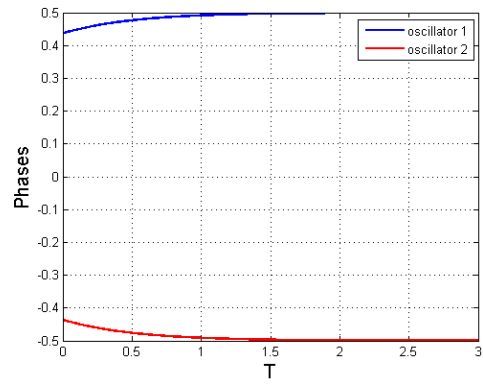

(a) Two oscillators, stable equilibrium

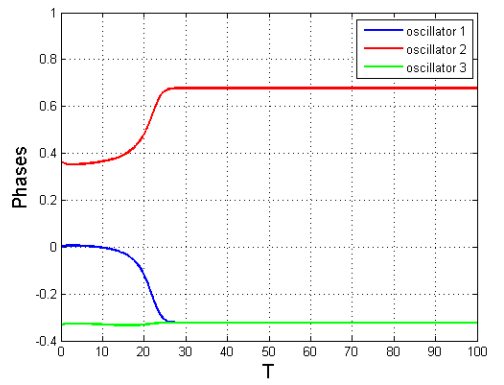

(b) Three oscillators, unstable equilibrium

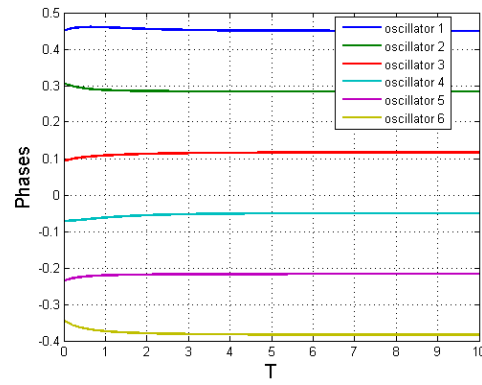

(c) Six oscillators, stable equilibrium

Fig. 5: Behavior of a system with two oscillators after a small perturbation from the stable anti-phase equilibrium (left), and of a system with three oscillators after a small perturbation from an unstable equilibrium.

5a. This equilibrium is stable, and the system converges to it after a perturbation.

\section{B. Three Oscillators}

Here we examine stability of equilibria of system (2) with three all-to-all connected oscillators. In that case the underlying topology is not a tree and we will employ Theorems 2 and 3 to show stability or instability. We will assume for simplicity that $\alpha_{i j}=1$ for all $i j \in E$.

The in-phase equilibrium is stable since condition (23) is satisfied: $\cos ^{2}(0)>\sin ^{2}(0)$. Clearly, the anti-phase equilibrium, i.e. when $\phi_{i}=\phi_{j}$ and $\phi_{k}=\phi_{i}+\pi$, is also stable.

Now consider equilibrium $\phi_{j}=\phi_{i}+2 \pi / 3, \phi_{k}=\phi_{i}-2 \pi / 3$, and a two-edge cut $C(P)=\{i j, i k\}$. Because $2\left(\cos ^{2}(2 \pi / 3)-\right.$ $\left.\sin ^{2}(2 \pi / 3)\right)<0$, condition (9) is satisfied and equilibrium is unstable.

Next equilibrium is defined as $\phi_{i}=\phi_{j}, \phi_{k}=\phi_{i}+\pi / 2$. Using cut $C(P)=\{k i, k j\}$, we obtain: $2\left(\cos ^{2}(\pi / 2)-\right.$ $\left.\sin ^{2}(\pi / 2)\right)<0$, which means that equilibrium is unstable due to the Theorem 2 .

If equilibrium is described by $\phi_{j}=\phi_{i}+\pi / 3, \phi_{k}=\phi_{i}-\pi / 3$, then for cut $C(P)=\{i j, i k\}: 2\left(\cos ^{2}(\pi / 3)-\sin ^{2}(\pi / 3)\right)<$ 0 , and equilibrium is unstable. On Fig. $5 b$ behavior of this system is shown after a small perturbation from this unstable equilibrium.

Finally, when $\phi_{j}=\phi_{i}+\pi / 2, \phi_{k}=\phi_{i}-\pi / 2$, and cut $C(P)=\{i j, i k\}: 2\left(\cos ^{2}(\pi / 2)-\sin ^{2}(\pi / 2)\right)<0$, equilibrium is unstable.

\section{Four Oscillators}

We will check that equilibrium of system (2) with four oscillators shown on Fig. 4 is unstable. At this equilibrium $\phi_{j}=\phi_{i}+\pi / 2, \quad \phi_{k}=\phi_{i}+\pi / 4, \phi_{l}=$ $\phi_{i}-\pi / 4$, and we define cut $C(P)$ as $C(P)=$ $\{i j, i k, i l\}$. Since $\left(\cos ^{2}(\pi / 2)-\sin ^{2}(\pi / 2)\right)+\left(\cos ^{2}(\pi / 4)-\right.$ $\left.\sin ^{2}(\pi / 4)\right)+\left(\cos ^{2}(\pi / 4)-\sin ^{2}(\pi / 4)\right)<0$, then Theorem 2 guarantees that this equilibrium is unstable.

\section{Six Oscillators}

In [28] it was shown that when in system (2) all coupling functions are $\sin ()$ and the underlying topology is a complete

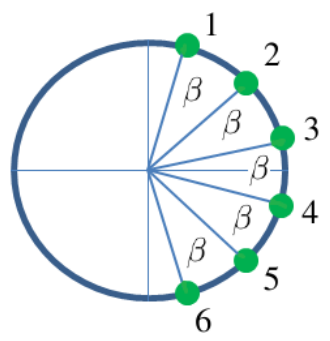

Fig. 6: Stable equilibrium corresponding to the example with six oscillators with $\beta=\pi / 6$.

graph, then the only stable equilibria are those in which every phase difference is a multiple of $\pi$. Using an example with six oscillators and $\sin ()$ coupling functions, we demonstrate that this property does not generally hold in case of an arbitrary topology.

In this example the underlying topology is a ring graph, so that the pairs of connected oscillators are $(1,2),(2,3),(3,4)$, $(4,5),(5,6),(6,1)$. The equilibrium is defined as follows: $\phi_{1}=\frac{5 \pi}{12}, \phi_{2}=\frac{\pi}{4}, \phi_{3}=\frac{\pi}{12}, \phi_{4}=-\frac{\pi}{12}, \phi_{5}=-\frac{\pi}{4}, \phi_{6}=-\frac{5 \pi}{12}$, $K_{12}=K_{23}=K_{34}=K_{45}=K_{56}=\cos \left(\frac{\pi}{6}\right), K_{16}=\cos \left(\frac{5 \pi}{6}\right)$. The phases of oscillators at the equilibrium are shown on Fig. 6. Using Theorem 3, it is easy to verify that this equilibrium is stable, and on Fig. 5c a behavior of the system is shown after a small perturbation from this equilibrium.

\section{CONCLUSION}

In this work we studied a model of arbitrary interconnected homogeneous coupled oscillators with a time-varying coupling. We demonstrated that systems of oscillators described by this model always achieve frequency synchronization. Sufficient stability and instability conditions for equilibria were provided for a general underlying topology, and a criterion of stability was formulated for a tree topology. Additionally, for the tree topology a condition on the coupling function was found that guarantees almost surely convergence to a stable equilibrium. We illustrated our theoretical results with several examples. 


\section{ACKNOWLEDGMENT}

The research is supported by ONR under N00014-12-11055 .

\section{REFERENCES}

[1] Aoki, Takaaki, and Toshio Aoyagi. "Co-evolution of phases and connection strengths in a network of phase oscillators." Physical review letters 102.3 (2009): 034101

[2] Aoki, Takaaki, and Toshio Aoyagi. "Self-organized network of phase oscillators coupled by activity-dependent interactions." Physical Review E 84.6 (2011): 066109.

[3] Bollobás, Béla. Modern graph theory. Vol. 184. Springer, 1998.

[4] Bressloff, P. C., and S. Coombes. "Travelling waves in chains of pulsecoupled integrate-and-fire oscillators with distributed delays." Physica D: Nonlinear Phenomena 130.3 (1999): 232-254.

[5] Brown, Eric, Philip Holmes, and Jeff Moehlis. "Globally coupled oscillator networks." Perspectives and Problems in Nolinear Science. Springer New York, 2003. 183-215.

[6] Cumin, D., and C. P. Unsworth. "Generalising the Kuramoto model for the study of neuronal synchronisation in the brain." Physica D: Nonlinear Phenomena 226.2 (2007): 181-196.

[7] Dörfler, Florian, and Francesco Bullo. "Synchronization in complex networks of phase oscillators: A survey." Automatica (2014).

[8] Dörfler, Florian, Michael Chertkov, and Francesco Bullo. "Synchronization in complex oscillator networks and smart grids." Proceedings of the National Academy of Sciences 110.6 (2013): 2005-2010.

[9] Gushchin, Andrey, Enrique Mallada, and Ao Tang. "Synchronization of Heterogeneous Kuramoto Oscillators with Arbitrary Topology." arXiv preprint arXiv:1410.7448 (2014)

[10] Seung-Yeal Ha, Zhuchun Li, Xiaoping Xue, Formation of phaselocked states in a population of locally interacting Kuramoto oscillators, Journal of Differential Equations, Volume 255, Issue 10, 15 November 2013, Pages 3053-3070, ISSN 0022-0396, http://dx.doi.org/10.1016/j.jde.2013.07.013.

[11] Hebb, Donald. "0.(1949) The organization of behavior." (1968): 44

[12] Hoppensteadt, Frank Charles, and Eugene M. Izhikevich. Weakly connected neural networks. Vol. 126. New York: Springer, 1997.

[13] Hoppensteadt, Frank C., and Eugene M. Izhikevich. "Oscillatory neurocomputers with dynamic connectivity." Physical Review Letters 82.14 (1999): 2983.

[14] Isakov, A., and L. Mahadevan. "Synchronization in a stochastic Hebbian network of phase oscillators." arXiv preprint arXiv:1404.2328 (2014).

[15] Kazantsev, Victor, and Alexey Pimashkin. "Forced phase-locked states and information retrieval in a two-layer network of oscillatory neurons with directional connectivity." Physical Review E 76.3 (2007): 031912.

[16] Khalil, Hassan K., and J. W. Grizzle. Nonlinear systems. Vol. 3. Upper Saddle River: Prentice hall, 2002.

[17] Kiss, István Z., Yumei Zhai, and John L. Hudson. "Emerging coherence in a population of chemical oscillators." Science 296.5573 (2002): 16761678.

[18] Kuramoto, Yoshiki. "Self-entrainment of a population of coupled nonlinear oscillators." International symposium on mathematical problems in theoretical physics. Springer Berlin Heidelberg, 1975.

[19] LaSalle, J.P. Some extensions of Liapunov's second method, IRE Transactions on Circuit Theory, CT-7, pp. 520527, 1960.

[20] Maistrenko, Yuri L., et al. "Multistability in the Kuramoto model with synaptic plasticity." Physical Review E 75.6 (2007): 066207.

[21] Mallada, Enrique, and Ao Tang. "Synchronization of phase-coupled oscillators with arbitrary topology." American Control Conference (ACC), 2010. IEEE, 2010

[22] Mallada, Enrique, and Ao Tang. "Synchronization of weakly coupled oscillators: coupling, delay and topology." Journal of Physics A: Mathematical and Theoretical 46.50 (2013): 505101.

[23] Mallada, Enrique, and Ao Tang. "Distributed clock synchronization: Joint frequency and phase consensus." Decision and Control and European Control Conference (CDC-ECC), 2011 50th IEEE Conference on. IEEE, 2011

[24] Marvel, Seth A., and Steven H. Strogatz. "Invariant submanifold for series arrays of Josephson junctions." Chaos: An Interdisciplinary Journal of Nonlinear Science 19.1 (2009): 013132.
[25] Moioli, Renan C., Patricia A. Vargas, and Phil Husbands. "The dynamics of a neural network of coupled phase oscillators with synaptic plasticity controlling a minimally cognitive agent." Artificial Neural NetworksICANN 2010. Springer Berlin Heidelberg, 2010. 245-255.

[26] Niyogi, Ritwik K., and L. Q. English. "Learning-rate-dependent clustering and self-development in a network of coupled phase oscillators." Physical Review E 80.6 (2009): 066213.

[27] Ren, Quansheng, and Jianye Zhao. "Adaptive coupling and enhanced synchronization in coupled phase oscillators." Physical Review E 76.1 (2007): 016207.

[28] Scardovi, Luca. "Clustering and synchronization in phase models with state dependent coupling." Decision and Control (CDC), 2010 49th IEEE Conference on. IEEE, 2010.

[29] Seliger, Philip, Stephen C. Young, and Lev S. Tsimring. "Plasticity and learning in a network of coupled phase oscillators." Physical Review E 65.4 (2002): 041906.

[30] Timms, Liam, and Lars Q. English. "Synchronization in phase-coupled Kuramoto oscillator networks with axonal delay and synaptic plasticity." Physical Review E 89.3 (2014): 032906.

[31] Winfree, Arthur T. "Biological rhythms and the behavior of populations of coupled oscillators." Journal of theoretical biology 16.1 (1967): 1542.

[32] Yamaguchi, Shun, et al. "Synchronization of cellular clocks in the suprachiasmatic nucleus." Science 302.5649 (2003): 1408-1412. 